\title{
Expression of LEKTI correlates with PNI and LVI in SCC of the oral tongue and mechanism of LEKTI loss in HNSCC
}

\begin{abstract}
Expression of Lympho-Epithelial Kazal-Type-Inhibitor (LEKTI), a broad spectrum protease inhibitor, is dysregulated in head and neck squamous cell carcinomas (HNSCC) and HNSCC cell lines. Here, we investigated expression of LEKTI in primary tumor specimens of 81 patients with SCC of the oral tongue in correlation with pathologic findings and clinical outcomes. IHC analyses have shown that LEKTI expression is negative in 31, intermediate in 44, and strong in 6 patients. Correlative analyses between LEKTI expression and perineural Invasion (PNI) and lymphovascular invasion (LVI) demonstrated that the relative risk of PNI and LVI were $3.2(95 \% \mathrm{CI}, 1.2$ to $8.9, \mathrm{p}=0.007$ by Chi Square Test) and $6.0(95 \%$ CI 1.2 to $40.8, p=0.01$ by Fisher's Exact Test) respectively in patients with LEKTI-negative tumors compared to those with LEKTI-positive tumors. Kaplan-Meier estimates showed that patients with LEKTI-negative expression had a $20 \%$ increased risk of disease recurrence (HR 1.19 and $95 \% \mathrm{CI} 0.61$ to 2.33 , $\mathrm{p}=0.23$ by Log rank test) and an $80 \%$ increased risk of death from all causes (HR 1.78 and $95 \%$ CI 0.34 to $9.41, p=0.48$ by Log rank test). Analysis of the covariates for disease recurrence and death in tongue cohort found no significant differences in age, gender, $\mathrm{T}$-stage, grade, $\mathrm{N}$-stage, and postoperative treatment between patients with LEKTI-negative and LEKTI-positive tumors. Further, we present evidence that transcriptional regulation is a very likely mechanism accounting for loss of LEKTI mRNA and protein from HNSCC. These data confirm our previous in vitro and orthotopic model of tongue cancer findings and shed new light on mechanisms of PNI and LVI in HNSCC.
\end{abstract}

Volume 2 Issue 5 - 2015

\author{
Thomas Shellenberger,' Mitchell J Frederick,' \\ Ying Henderson,' Yaan Kang,' Karthik \\ Jayakumar, ${ }^{2}$ Rena Janarthanan, ${ }^{3}$ Gary L \\ Clayman, ${ }^{\prime}$ Rajendra Prasad, ${ }^{4}$ Arumugam \\ Jayakumar ${ }^{1,4,5}$ \\ 'Department of Head and Neck Surgery, The University of Texas \\ MD Anderson Cancer Center, USA \\ ${ }^{2}$ Department of Medicine, Baylor College of Medicine, USA \\ ${ }^{3}$ The American University of Antigua College of Medicine, \\ Antigua and Barbuda \\ ${ }^{4}$ Amity Institute of Integrative Sciences and Health,Amity \\ University of Haryana, India \\ ${ }^{5}$ Department of Experimental Therapeutics, The University of \\ Texas MD Anderson Cancer Center, USA
}

Correspondence: Department of Experimental Therapeutics, The University of Texas MD Anderson Cancer Center, USA, Email ajayakum@mdanderson.org

Received: October 23, 2015 | Published: November 09, 2015

Keywords: SPINK5, LEKTI, lymphovascular invasion, perineural invasion, HNSCC, fisher's exact test

Abbreviations: LEKTI, lympho-epithelial kazal-typeinhibitor; SPINK5, serine protease inhibitor kazal-type 5; HNSCC head and neck squamous cell carcinoma; PNI, perinural invasion; LVI, lymphovascular invasion

\section{Introduction}

Local tumor invasion including perinural invasion (PNI) and lymphovascular invasion (LVI) occur by the attachment of tumor cells to components of the extracellular matrix (ECM) and by degradation of ECM by proteinase enzymes elaborated into the tumor microenvironment. ${ }^{1-6}$ These processes are regulated by such proteolytic enzymes as serine proteases, cysteine proteases, and matrix metalloproteinases (MMPs) tightly balanced by their endogenous inhibitors in the tumor microenvironment. ${ }^{7-13}$ Thus, inhibition of such proteinases can disrupt critical steps of invasion and metastasis. Indeed, several proteinase inhibitors have shown importance in a range of cancer types by the loss of expression correlating with advanced tumor progression. ${ }^{14-19}$ Moreover, proteinase inhibitors have been demonstrated to hold tumor suppressor functions that oppose tumorigenesis, proliferation, angiogenesis, invasion, and metastasis. ${ }^{20-22}$

An inhibitor of multiple serine proteinases, lymphoepithelial kazaltype inhibitor (LEKTI), was identified and cloned in our laboratory on the basis of its constitutive expression in normal oral mucosa and loss of its expression in matched head and neck squamous cell carcinoma (HNSCC) specimens and multiple HNSCC lines. ${ }^{23}$ Recently it was confirmed in a sub-set of HNSCC. ${ }^{24}$ It was also shown by several investigators that LEKTI protein was encoded by SPINK5 gene and mutations in SPINK5 has been linked to the inherited disorder known as Netherton Syndrome. ${ }^{25-41}$ We produced recombinant full length LEKTI and several of its fragments using baculovirus expression system and established that recombinant human LEKTI inhibits a battery of serine proteinases in vitro including plasmin, trypsin, cathepsin G, human KLKs, and elastase, enzymes implicated in the activation ofmMPs. ${ }^{42-47}$ Recently, we demonstrated that stable expression of LEKTI in HNSCC OSC-19 cells resulted in markedly decreased levels of expression of genes encodingmMP-9,mMP-14, KLK5, and ADAM8. Furthermore, LEKTI over expressing cells displayed striking morphological changes are more adhesive and less invasive, ${ }^{48}$ These results demonstrate a novel negative regulatory role for LEKTI in modulating the production of keymMPs involved in ECM degradation and suggest that loss of LEKTI in HNSCC tumor cells could have a pivotal role in HNSCC progression. Subsequently, we determined the consequences of LEKTI re-expression on the in vivo changes in the tumor growth and invasion using an orthotopic model of tongue cancer. ${ }^{49}$ We have demonstrated that in the tongue tumors of mice, lymphovascular invasion or perineural spread was found in $100 \%$ of tumors derived from vector or parental cell lines but was almost totally absent in all tumors derived from LEKTIexpressing clones. Thus, we hypothesized that loss of LEKTI expression in primary tumors correlates with aggressive biologic behavior in patients with HNSCC. To test our hypotheses, we now investigated the expression of LEKTI in primary tumor specimens of 
patients with SCC of the oral tongue in correlation with pathologic findings and clinical outcomes and also the mechanism of its loss. Altogether, our results showed a heterogeneous expression of LEKTI, an association between LEKTI expression and occurrence of PNI and LVI and an epigenetic mechanism of regulation in SCC of the oral tongue.

\section{Materials and methods}

\section{Materials}

The following reagents were obtained commercially as indicated: Erase-a Base nested deletion mutagenesis system, luciferase reporter plasmid pGL3-Basic and Dual luciferase assay kit (Promega Corp., Madison, WI); pCRII-TOPO (Life Technologies, Rockville, MD); Genomic DNA isolation kit (Qiagen, Valencia, CA ); sodium bisulfite, hydroquinone, sodium acetate, 5'-azacytidine (Sigma-Aldrich, St. Louis, MO); primary normal human epidermal keratinocytes (NHEKs) and keratinocyte growth medium (Cambrex Biosciences, Walkersville, MD); precast sodium dodecyl sulfate (SDS)polyacrylamide gels, prestained markers (Bio-Rad Laboratories, Hercules, CA); anti-LEKTI mAb 1C11G6 (Zymed Laboratories, San Francisco, CA); horseradish peroxidase-conjugated goat-anti-mouse IgG $(\mathrm{H}+\mathrm{L})$ (Jackson ImmunoResearch Laboratories, West Grove, PA) lipofectamine 2000 and pcDNA3.1 (-) (Invitrogen, Carlsbad, CA); ECL kit (Amersham Bioscience Corporation, Piscataway, NJ); Kodak X-AR5 films (Eastman Kodak, Rochester, NY); Sense and antisense oligonucleotides specific for human LEKTI were synthesized by Genosys Biotechnologies Inc. (The Woodlands, TX); restriction endonucleases and polymerase chain reaction reagents (New England Biolabs, Beverely, MA); UMSCC-1 is an established HNSCC tumor line derived from patients with squamous cell carcinoma of the floor of mouth.

\section{Cell culture and transfections}

The HNSCC cell line UMSCC-1 was obtained from Dr. Tom Carey at the University of Michigan. NHEKs were cultured in keratinocyte growth medium containing low-calcium. All cells were cultured at $37^{\circ} \mathrm{C}$ in humidified incubator with $5 \% \mathrm{CO} 2$ and $95 \%$ air. Cells are cotransfected in triplicate with $1 \mathrm{mg}$ reporter construct and $25 \mathrm{ng}$ Renilla luciferase or control vector plasmid DNA using Lipofectamine 2000 according to the manufacturer's instructions. Approximately 50\% transfection efficiency was achieved as determined by transfection with a GFP control plasmid.

\section{IHC of LEKTI protein expression in primary tumors}

The primary tumor specimens were recut and stained with a purified mouse anti-LEKTI monoclonal antibody. Specimens were stained for LEKTI with an automated stainer. Briefly, sections $(4 \mu \mathrm{m}$ thick) will be deparaffinized, dehydrated, antigen retrieved using microwave methods, immersed in $0.3 \% \mathrm{H} 2 \mathrm{O} 2$ for $10 \mathrm{~min}$ at room temperature in methanol, and washed with PBS. The sections will be incubated with $3 \%$ BSA in PBS, washed twice, and then incubated with a mouse monoclonal anti-PCNA antibody (Dako) for $1 \mathrm{~h}$ at room temperature. Primary antibody will be detected with a Vectastain ABC-alkaline phosphatase kit (Vector Laboratories, Burlingame, CA). Two investigators will examine the stained specimens independently. In each case, three arbitrary separate microscope fields $(\times 200)$ will be examined to count immunoreactive tumor cells and the total number of tumor cells. The average percentage of immunostained cells will be defined as the labeling index (LI) and used for statistical analysis. The1C11G6 mAb, which is specific for LEKTI, will be detected using the Vectastain ABC-alkaline phosphatase kit according to supplier's instructions. Slides were reviewed and categorized by two independent investigators. LEKTI scoring was done as follows: Tumors with weak to no LEKTI staining in $>95 \%$ cells were considered negative; Tumors with moderate LEKTI staining in $>5 \%$ of cells or strong staining restricted to more differentiated tumor were considered intermediate; Tumors with strong LEKTI staining in $>5 \%$ of cells, not restricted to more differentiated areas were considered strongly positive. The surgical pathology reports were reviewed for histopathologic features of the primary tumor. Medical records were reviewed for covariate and clinical follow-up data. Time-to-event analysis was performed with the Kaplan-Meier method for patients stratified by LEKTI-staining pattern.

\section{Luciferase reporter assays}

A series of 5' deletion mutants derived from the 343bp LEKTI promoter is generated using the Erase-a Base nested deletion mutagenesis system and cloned into the luciferase reporter plasmid pGL3-Basic. To control for efficiency of transfection, the pRL-CMV vector containing Renilla luciferase driven by the CMV enhancer/ promoter is co-transfected with constructs derived from pGL3-Basic. The products of firefly and Renilla luciferase can be distinguished from each other using the Dual-luciferase Reporter Assay. Cells (4 $\times 105)$ are seeded in 6-well plates the day before transfection. On the day of transfection, $3 \mu \mathrm{g}$ DNA from each construct and $0.125 \mu \mathrm{g}$ pRL-CMV (internal control) is transfected into each well with $3 \mu \mathrm{l}$ Lipofactamine 2000. After incubating at $37^{\circ} \mathrm{C}$ for $4 \mathrm{~h}$, complete medium is added and cells are harvested $40 \mathrm{~h}$ after transfection by scraping off into lysis buffer $(500 \mu 1)$ as described in the Dual luciferase assay kit. After storing at $-80^{\circ} \mathrm{C}$ for overnight, cell extracts are cleared by centrifugation for $2 \mathrm{~min}$ at $4^{\circ} \mathrm{C}$ and $20 \mu \mathrm{l}$ are used for luciferase assay. The luciferase activity is measured with the dual-luciferase reporter assay system by a micro titer plate luminometer (Dynex Revelation 4.06, Dynex Technologies, Chantilly, VA) according to the manufacturer's protocol. Transfections are performed in triplicate wells.

\section{Primer extension}

An antisense oligonucleotide approximately $100 \mathrm{bp}$ downstream of the predicted start of site of transcription is synthesized, labeled with T4 polynucleotide kinase and $[\gamma-32 \mathrm{P}]$ ATP, hybridized to $20 \mathrm{mg}$ total RNA from NOE cells, and extended by AMV reverse transcriptase (in the presence of actinomycin $\mathrm{D}$ ). The extended DNA product is treated with RNase, ethanol precipitated and loaded onto a $9 \%$ acrylamide $/ 7 \mathrm{M}$ urea sequencing gel. To pinpoint the start of transcription, a $500 \mathrm{bp}$ fragment of genomic DNA containing the primer extension binding site and upstream genomic sequences is cloned ahead of time into a plasmid vector, and cycle sequenced with the same antisense oligonucleotide used in the primer extension experiment. The primer extension product is loaded alongside the sequencing reactions for comparison.

\section{Northern blot and real time PCR}

Total RNA isolation, Northern blot and Real-time PCR were performed as described earlier. ${ }^{28}$ Total RNA was prepared using TriZol reagent (Invitrogen) according to the manufacturer's instructions. For Northern blot, $20 \mu \mathrm{g}$ total RNA was applied to a $1 \%$ formaldehyde agarose gel. After transferring RNA to Hybond-Np membrane (Amersham), the membrane and the filter were hybridized with 32P-r- 
human LEKTI or 32P-GAPDH. For Real-time PCR, $2 \mu \mathrm{g}$ total RNA were reverse transcribed (RT) by Superscript II (Life Technologies) in a $25 \mu 1$ total reaction volume containing RT buffer, random hexamers, dNTP, and RNase inhibitor (Roche Applied Science, Indianapolis, IN). Real-time PCR was performed in a $25 \mu 1$ total reaction volume containing $1 \mu$ of $1: 10$ diluted cDNA obtained from RT reaction, $12.5 \mu 1$ of TaqMan Universal PCR Master Mix without AmpErase UNG, and $1.25 \mu$ of specific primers for LEKTI gene on ABI Prism $7900 \mathrm{HT}$. As a control, $18 \mathrm{~S}$ primers were used, and cDNA was diluted to 1:500. Serial dilutions of the standard templates were also used for parallel amplifications. Levels of LEKTI mRNA were normalized to those of $18 \mathrm{~S}$ in each sample.

\section{Nuclear extracts isolation and DNAse i footprinting assays}

Cells are scraped in cold PBS, centrifuged, and suspended in buffer A $(10 \mathrm{mM}$ HEPES, $10 \mathrm{mM} \mathrm{KCl}, 0.1 \mathrm{mM}$ EDTA, $0.1 \mathrm{mM}$ EGTA, 1mM DTT and 0.5mM PMSF, pH 7.9). After 15min on ice, cells are centrifuged and nuclear pellets disrupted in cold buffer B (20mM HEPES, $0.4 \mathrm{M} \mathrm{NaCl}, 1 \mathrm{mM}$ EDTA, 1mM EGTA, $1 \mathrm{mM}$ DTT, $1 \mathrm{mM}$ PMSF and $20 \%$ glycerol, PH 7.9 ) by homogenizing with 10 strokes and incubating further for $30 \mathrm{~min}$ at $4{ }^{\circ} \mathrm{C}$. After an additional centrifugation, the supernatant containing nuclear extracts is harvested. DNase I footprinting is carried out using Promega's core footprinting kit with modifications. Probes are PCR generated, subcloned into the pBlueTOPO vector (Invitrogen), excised with HindIII/BamHI, gel purified using a QIAEX II Gel Extraction Kit (Qiagen), and labeled with [32P] ATP using T4 polynucleotide kinase. Labeled probe is digested with either HindIII or BamHI to achieve selective labeling of either sense or anti-sense strands. Increasing amounts of nuclear extract is incubated with labeled probe $(10,000 \mathrm{cpm})$ at room temperature for $10 \mathrm{~min}$, followed by digestion with 6 units of DNase I at room temperature for $3 \mathrm{~min}$. Digested products is phenol/chloroform extracted, ethanol precipitated, and analyzed using $6 \%$ acrylamide sequencing gels containing $8 \mathrm{M}$ urea. For comparison, DNA-sequence reactions of the genomic DNA using a proximal primer are run in parallel.

\section{Microdissection, DNA extraction, and LOH}

Consecutive 5-micron sections from formalin fixed paraffin embedded specimens are cut and placed onto positively charged slides. One slide is stained with H\&E, and another with Methyl Green. Areas of tumor or normal identified on H\&E slide are used to localize corresponding regions on Methyl-Green stained slide to be microdissected. A number 15 scalpel blade is dunked into PCR/ PK buffer containing $0.5 \%$ Tween 20 and solution transferred to area that is to be scraped. Approximately 100 to 200 cells are scraped into puddle of PCR/PK buffer and transferred to a PCR tube containing $15 \mu \mathrm{l} \mathrm{PCR} / \mathrm{PK}$ buffer plus Proteinase K $(0.4 \mathrm{mg} / \mathrm{ml})$. Digestion reactions are overplayed with mineral oil and incubated at $55^{\circ} \mathrm{C}$ with shaking overnight. Fresh Proteinase $\mathrm{K}$ is added and the overnight incubation repeated two more times. Proteinase $\mathrm{K}$ is heat inactivated by incubation for $15 \mathrm{~min}$ at $95^{\circ} \mathrm{C}$, and $5 \mu$ digested DNA is used per PCR reaction. For LOH analysis, DNA is amplified using Taq Polymerase Gold (Perkin Elmer) for 37 cycles in the presence of fluorescently labeled primer. The size of PCR products is then compared on an automated DNA sequencer. In some cases, DNA is extracted from leukocytes using DNAzol (Molecular Research Products) according to the manufacturer's instructions. Primer sets for the two polynucleotide repeat regions within the LEKTI locus are: (1) 5'CACAAACATACCAATTTTAACAAACC-3'(sense) and 5'-
TGAACCACATCGCTATTAAGAGTTTT-3'(antisense); and (2) 5'-CAGCCTGGGCAACAGAGTAA-3'(sense) and 5'-CACCACTGAATAGATTTTAGGGAGG-3'(antisense).

\section{Bisulfite treatment and LEKTI promoter methylation}

The methylsation patterns in LEKTI promoter region was determined as described in the literature ${ }^{50}$ Genomic DNA from NHEK and UMSCC1 cells $(2 \mu \mathrm{g})$ was isolated and treated with bisulfite according to the published protocol. ${ }^{50}$ Bisulfite-treated genomic DNA $(0.2 \mu \mathrm{g})$ was used in PCR using bisulfite PCR primers designed by MethPrimer software from University of California San Francisco. PCR products were subcloned into pCRII-TOPO (Life Tech.), ten clones were selected randomly, and sequenced by Core facility in MD Anderson Cancer Center.

\section{Statistical analysis}

Fisher's exact test was used to examine associations between LEKTI expression and PNI and LV in human samples and clinical variables. Kaplan-Meier survival curves and log rank tests were used to examine the association between tumor expression of LEKTI and patient disease-specific survival. Multivariable analysis was performed using the Cox proportional hazards model. The data for this analysis were tested (plot of differences in log of cumulative hazard rates of test variable against time) and found to conform to the proportional hazards assumptions. Data are presented as mean $\pm \mathrm{SD}$. Statistical comparisons between experimental groups were analyzed by unpaired Student's $t$ test, and a two-tailed $p<0.05$ was taken to indicate statistical significance. For analyzing the correlation, we used Pearson's test and the $\mathrm{p}$ values are indicated. We also used Mann-Whitney rank sum test as indicated. All statistical tests are two-sided. Differences in variables that were not normally distributed were compared using a nonparametric test (Mann-Whitney U test). Again, a p value less than 0.05 is deemed statistically significant. All statistical tests are two-sided.

\section{Results and discussion}

\section{LEKTI expression correlates with perinuclear invasion (PNI) in patients with SCC of the tongue}

We previously published that both LEKTI mRNA and LEKTI protein was clearly absent from the HNSCC lines UMSCC-1, OSC19, Tu-138, JMAR, and DM14 compared to abundant message and protein in normal oral epithelial cells. ${ }^{48,50}$ To characterize the expression of LEKTI in primary tumors, we performed immunohistochemical analysis of paraffin-embedded, formalin-fixed specimens from 60 unselected patients with HNSCC from various primary sites and stages. During initial review of unselected HNSCC specimens for LEKTI expression by IHC, PNI was incidentally noted in 3 cases. In all three cases the tumors were negative for LEKTI expression, including tumor cells that invaded the perineural spaces. Photomicrographs from one of these cases showing a crescent pattern of PNI are presented (Figure 1 top panel). Although the correlation between LEKTI status and PNI invasion has not been systematically studied in the sample group, the incidental finding of reduced expression in tumor cells mediating PNI is consistent with the hypothesis generated from animal experiments in which LEKTI expression was correlated with reduced PNI.

Based on these anecdotal findings and our results from animal experiments, we investigated the relationship between LEKTI expression and PNI in a preliminary study using a more homogenous cohort of patients. The MDACC Tumor Registry was used to identify a 
cohort of 81 consecutive, previously untreated patients who underwent surgery as initial treatment for SCC of the oral tongue between 1990 and 1993. Expression of LEKTI in tumors from patients of this cohort was examined by IHC (using an automated stainer) and evaluated by two independent investigators, including a board-certified head and neck pathologist. LEKTI staining of tumors was evaluated as either negative, intermediate, or strong according to criteria described in methods section. A representative photomicrograph showing these three patterns are shown (Figure 1, bottom panel). When we analyzed the entire cohort for LEKTI expression we observed that LEKTI expression was negative in $38 \%(31 / 81)$, intermediate in $54 \%(44 / 81)$, and strong in $7.4 \%(6 / 81)$ of samples (Table 1). The distribution of staining patterns resembled that previously observed in unselected cases, demonstrating that the expression patterns can be reliably categorized.

Figure 1
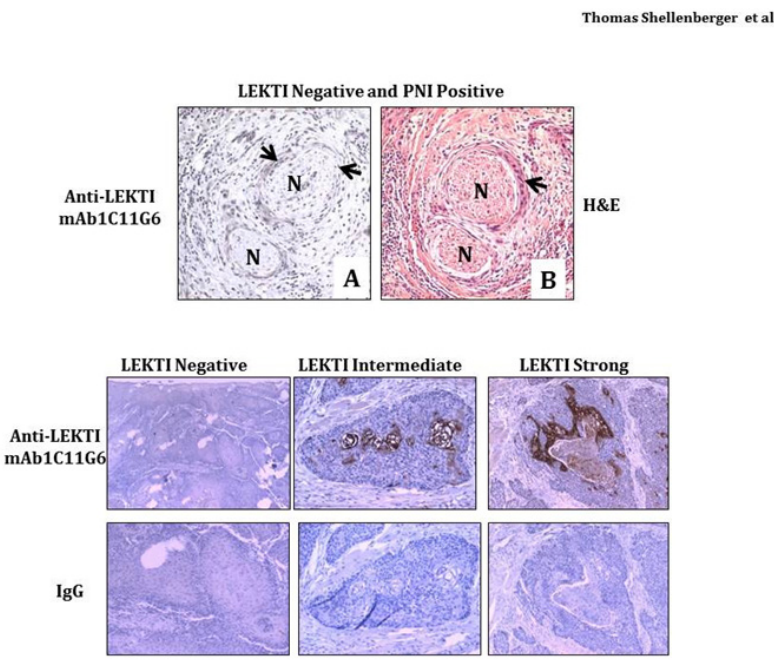

Figure I Characterization of LEKTI expression and PNI in tumor specimens from squamous cell carcinoma (SCC) of the tongue.

Top panel: A tumor biopsy specimen from an unselected HNSCC specimen was examined for LEKTI expression by immunohistochemistry $(A)$ and the corresponding region was also stained by H\&E on a parallel section (B). In the first view, a crescent pattern of $\mathrm{PNI}$ is apparent $(\mathrm{A})$, and LEKTI expression is absent from tumor cells invading the perineural space (A). Arrow heads point to tumor nests, and nerve segments are denoted with an "N".

Bottom panel: SCC tongue specimens were examined for LEKTI protein by IHC. Immunostaining was performed in with an automated staining machine (Dako) using an LSAB+ kit. Incubation of the primary antibody was performed with anti-LEKTI diluted at I:I50 for 60 minutes. Slides were analyzed by light microscopy.A typical example of each petern of LEKTI expression was shown. LEKTI expression was negative in $3 \mathrm{I}$, intermediate in 44, and strong in 6 patients.

The surgical pathology report of each patient from the cohort was then retrospectively reviewed for histopathological features of the primary tumor. The number of LEKTI tumors with each pattern of expression as a function of PNI status is also included in Table 1. PNI was present in $37 \%$ (i.e.12/31) of patients with LEKTInegative tumors. Conversely, PNI was present in just $11 \%$ (i.e., $5 / 44$ ) of patients with LEKTI-intermediate tumors and $16 \%$ (i.e. 1/6) of patients with tumors staining strongly for LEKTI. While a statistically significant difference in PNI status $(\mathrm{P}=0.01$, Fisher's Exact test $)$ was found between tumors negative for LEKTI and those with intermediate expression, the small number of patients in the LEKTIstrong group limited meaningful comparison (Table 1). Therefore, PNI was compared between patients with LEKTI-negative staining and patients with LEKTI-positive staining, whether intermediate or strong (Table 2). PNI was present in $37 \%(12 / 31)$ of patients with LEKTI-negative tumors. In contrast, PNI was present in just $12 \%$ (6/ 50) of patients with LEKTI-positive tumors. Therefore, in patients with LEKTI-negative tumors, the relative risk of PNI was 3.2 times greater $(95 \% \mathrm{CI}$ from 1.2 to 8.9$)$ than patients with LEKTI-positive expression ( $\mathrm{p}=0.007$ by Chi Square Test).

Table I Pattern of LEKTI expression in PNI (+) and PNI (-) tumors from cohort

\begin{tabular}{llll}
\hline LEKTI expression & PNI (+) & PNI (-) & Total \\
\hline Negative & 12 & 19 & 31 \\
Intermediate & 5 & 39 & 44 \\
Strong & 1 & 5 & 6 \\
Total & 18 & 63 & 81 \\
\hline
\end{tabular}

$\mathrm{PNI}$ is significantly different in LEKTI negative tumors compared to LEKTI intermediate tumors; $\mathrm{P}=0.0 \mathrm{l}$ (Fisher's Exact test)

$\mathrm{PNI}$ in LEKTI negative compared to LEKTI strong; $\mathrm{P}=0.39$ (Fisher's Exact test)

Table 2 PNI status of LEKTI positive and LEKTI negative tumors

\begin{tabular}{llll}
\hline LEKTI expression & PNI (+) & PNI (-) & Total \\
\hline Negative & 12 & 19 & 31 \\
Strong & 6 & 44 & 50 \\
Total & 18 & 63 & 81
\end{tabular}

$\mathrm{P}=0.007$ (Chi-square)

Relative risk $(95 \% \mathrm{Cl})$ of $\mathrm{PNI}$ in patients with LEKTI negative tumors compared to those with LEKTI positive tumors $=3.2$ (I.2 to 8.9$)$

\section{LEKTI expression correlates with lymphovascular invasion (LVI) in patients with SCC of the tongue}

In our cohort of 81 patients, the patterns of LEKTI expression were also compared with LVI of the primary tumor specimen. LVI was present in $23 \%(7 / 30)$ of patients with LEKTI negative tumors (Table 3). In contrast, LVI was present in just $4 \%(2 / 51)$ of patients with LEKTI-intermediate or -strong tumors. Therefore, in patients with LEKTI negative tumors, the relative risk of LVI was 6.0 times greater $(95 \%$ CI from 1.2 to 40.8 ) than patients with intermediate or strong LEKTI expression ( $\mathrm{p}=0.01$ by Fisher's Exact Test). These data in patients confirm our findings in the orthotopic tongue xenografts and support the foundation of this proposal because they strongly support that the loss of LEKTI expression in SCC of the oral tongue correlates with a locally aggressive biologic behavior. Kaplan-Meier estimates of disease-free survival and overall survival showed that patients with LEKTI-negative expression had a $20 \%$ increased risk of disease recurrence (HR 1.2 and $95 \%$ CI 0.61 to $2.33, \mathrm{p}=0.23$ by Log rank test) (Figure 2A) and an $80 \%$ increased risk of death from all causes (HR 1.8 and $95 \%$ CI 0.34 to $9.41, \mathrm{p}=0.48$ by Log rank test) (Figure 2B). In contrast, analysis of the covariates for disease recurrence and death found no significant differences in age, gender, T-stage, grade, $\mathrm{N}$-stage, and postoperative treatment between patients with LEKTI-negative and LEKTI-positive tumors (Table 4). 
Table 3 LVI status of LEKTI positive and LEKTI negative tumors

\begin{tabular}{llll}
\hline LEKTI expression & LVI (+) & LVI(-) & Total \\
\hline Negative & 7 & 23 & 31 \\
Strong & 2 & 49 & 50 \\
Total & 9 & 72 & 81 \\
\hline
\end{tabular}

$\mathrm{P}=0.0 \mathrm{I}$ (Fisher's Exact test)

Relative risk $(95 \% \mathrm{Cl})$ of $\mathrm{LVI}$ in patients with LEKTI negative tumors compared to those with LEKTI positive tumors $=6.0$ (I.2 to 40.8$)$

A

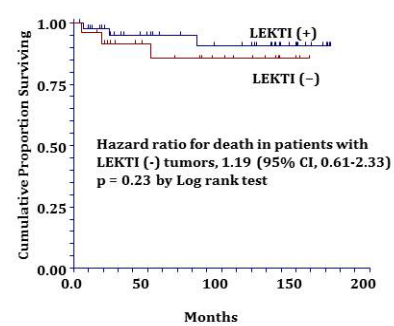

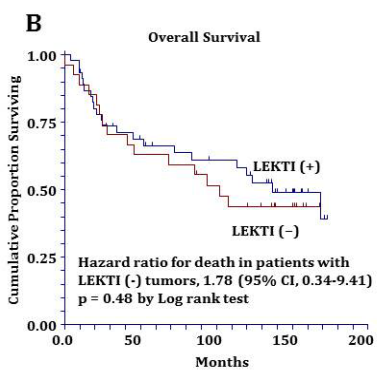

Figure 2 Disease-free survival and overall survival. Kaplan-Meier estimates of disease-free survival and the corresponding hazard ratio in the LEKTI (+) and LEKTI (-) group. Patients with LEKTI-negative expression had a $20 \%$ increased risk of disease recurrence (HR I.I 9 and $95 \% \mathrm{Cl} 0.6 \mathrm{I}$ to $2.33, \mathrm{p}=0.23$ by Log rank test) (A). Kaplan-Meier estimates of overall survival and the corresponding hazard ratio in the LEKTI (+) and LEKTI (-) group. Patients with LEKTI-negative expression had an $80 \%$ increased risk of death from all causes (HR I.78 and $95 \% \mathrm{Cl} 0.34$ to $9.4 \mathrm{I}, \mathrm{p}=0.48$ by Log rank test) (B).

Table 4 Analysis of the covariates for disease recurrence

\begin{tabular}{llll}
\hline & $\begin{array}{l}\text { LEKTI (-) } \\
\text { N=3 I }\end{array}$ & $\begin{array}{l}\text { LEKTI (+) } \\
\mathbf{N}=\mathbf{5 0}\end{array}$ & Comparison \\
\hline $\begin{array}{l}\text { Age(years) } \\
\begin{array}{l}\text { Gender( percent } \\
\text { male) }\end{array}\end{array}$ & 54.8 & 54.4 & $\mathrm{P}=0.87$ by t test \\
T stage(\%) TI & $13 \%$ & $32.20 \%$ & $\mathrm{P}=0.64$ by Chi Square \\
T2 & $30 \%$ & $39 \%$ & \\
T3 & $10 \%$ & $13 \%$ & $\mathrm{P}>0.05$ by Fisher's Exact \\
T4 & $13.30 \%$ & $4 \%$ & \\
cN stage(\%) cN0 & $22 \%$ & $43 \%$ & \\
cN+ & $14 \%$ & $27 \%$ & \\
$\begin{array}{l}\text { Post op treatment } \\
\text { (\%) Sx }\end{array}$ & $57 \%$ & $69 \%$ & \\
Sx+XRT & $43 \%$ & $29 \%$ & \\
Sx+ChemoXRT & $0 \%$ & $2 \%$ & \\
\hline
\end{tabular}

LOH is not a mechanism for loss of LEKTI mRNA and protein from HNSCC

In HNSCC biopsy specimens there appeared to be two patterns of LEKTI protein loss, with some tumors completely negative and others focally positive in areas where tumor cells were developing keratin pearls. Despite the fact that all established HNSCC tumor lines examined contained no LEKTI protein expression, some cell lines expressed mRNA. Together, these observations suggest multiple mechanisms for loss of LEKTI expression including both epigenetic and genetic abnormalities. The heterogeneous expression observed for LEKTI in tumor biopsies suggested an epigenetic mechanism of regulation. To further rule out the potential contribution of genetic loss, however, we analyzed 20 archival HNSCC biopsy specimens for $\mathrm{LOH}$ within the LEKTI locus using microsatellite markers found within the gene. Only $2 / 15$ (i.e., $13.3 \%$ ) of informative specimens had $\mathrm{LOH}$ within the LEKTI locus, making genetic deletion an unlikely mechanism.

\section{Promoter site methylation does not contribute to loss of LEKTI expression}

Epigenetic change has been recognized as an important underlying mechanism of genetic alteration in human malignancies, including HNSCC. Therefore, we examined the methylation status of the LEKTI promoter region in UMSCC1 and NHEK cell lines. The promoter region of LEKTI expressing cells of NHEK was extensively methylated in several of the $\mathrm{CpG}$ sites (Figure 3A). Similarly, the promoter region of non-expressing cells of UMSCC-1 was also sporadically methylated at several $\mathrm{CpG}$ islands (Figure 3B). These results suggest no correlation between LEKTI expression and methylation of its promoter site. To establish that methylation was not responsible for silencing LEKTI gene expression, cell lines with LEKTI promoter methylation was treated with 5'-azacytidine, a demethylating agent. In each of the two cell lines tested, 5-azaC did not alter LEKTI expression as shown by real-time PCR (Figure $3 \mathrm{C})$. These findings suggest that LEKTI expression is not regulated by promoter site methylation. We have not ruled out the mutational inactivation of LEKTI gene in HNSCC.

Figure 3

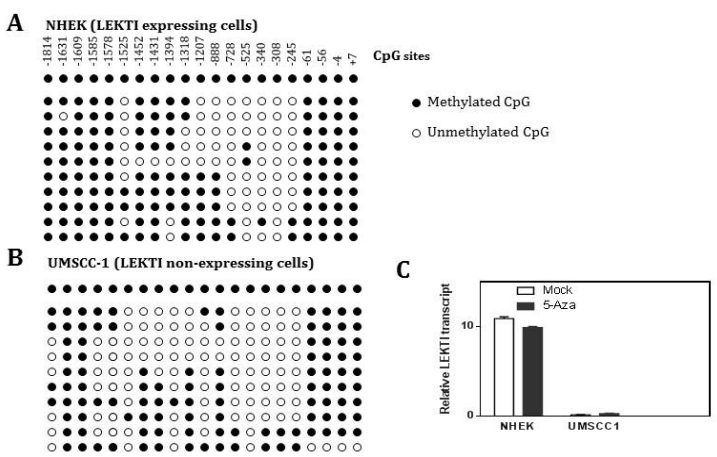

Figure 3 Characterization and assessment of LEKTI promoter activity. LEKTI promoter is active in LEKTI positive cell line NHEK but inactive in LEKTI negative cell line UMSCC-I (A). A series of promoter constructs ranging from -167bp to -1978bp upstream of the LEKTI transcription start site were cloned into luciferase reporter constructs. NHEK or UMSCC-I cells are co-transfected in triplicate with I mg reporter construct and $25 \mathrm{ng}$ Renilla luciferase. Cell lysates are prepared $36 \mathrm{~h}$ post transfection and the dual luciferase assay is used to measure firefly luciferase activity and normalize against values for Renilla luciferase. Values are divided by the mean value for the promoter less vector $\mathrm{PGL3}$, to calculate the relative LEKTI promoter activity (A). Protection of three distinct regions from the LEKTI promoter in a DNase I foot print assay (B).A labeled DNA probe spanning regions -1235 to - I546 of the LEKTI promoter was incubated with buffer alone (I), 5.8ug (2), $29 \mu \mathrm{g}$ (3), or $58 \mu \mathrm{gg}(4)$ of nuclear extract from UMSCC-I cells before digestion with DNase I. Regions A, B, C corresponds to three protected regions. 


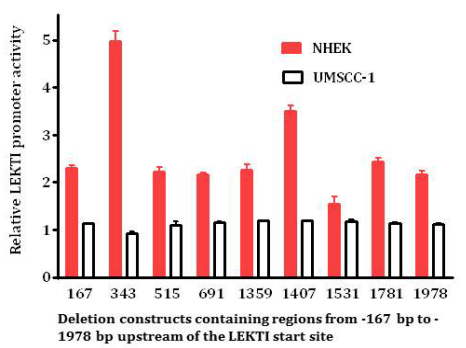

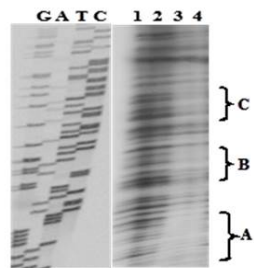

Figure 4 Silencing of LEKTI expression is not due to promoter methylation. A shows methylation patterns of individually sequenced clones of the LEKTI promoter region in NHEK cell line which shows LEKTI expression. B shows methylation patterns of individually sequenced clones of the LEKTI promoter region in HNSCC UMSCC-I tumor line which shows no LEKTI expression. $C$ shows that in each of the two cell lines tested, 5 -aza did not alter LEKTI expression.

\section{Transcriptional regulation accounts for loss of LEKTI mRNA and protein from HNSCC}

To begin addressing transcriptional mechanisms, we characterized the LEKTI promoter in primary cultures of NHEK. Primer extension analysis revealed a potential transcription start site (designated as $+1 \mathrm{bp}$ ) exactly $65 \mathrm{bp}$ upstream of the "ATG" start codon in exon 1. Inspection of the surrounding nucleotide sequence revealed a "TATAAAA" box 23bp upstream of the transcription start site. An identical transcriptional start site was predicted with the computer program Neural Network Promoter Prediction at: http://www. fruitfly.org/seq tools/promoter.html. A series of deletion constructs containing regions from -167bp to -1978bp upstream of the LEKTI start site were cloned in front of the firefly luciferase reporter gene in pGL3, and transiently transfected into NHEK (which express LEKTI) or UMSCC-1 (which do not express LEKTI). As shown in Figure 4A, the $343 \mathrm{bp}$ and $1407 \mathrm{bp}$ constructs exhibited 5- and 3-fold greater activity relative to pGL3 alone in NHEK, but none of the constructs were efficiently transcribed in the LEKTI negative cell line UMSCC-1. Regulation in NHEK appears complex, as there seems to be repressor sequences contained between $-343 \mathrm{bp}$ to $-515 \mathrm{bp}$, and between $-1407 \mathrm{bp}$ and $-1531 \mathrm{bp}$. Also, there appears to be an enhancer sequence present between -1359bp and -1407.

In preliminary experiments designed to detect possible repressor sequences in the LEKTI promoter, a $312 \mathrm{bp}$ probe spanning regions -1235 to -1546 was incubated with UMSCC-1 nuclear extract in a DNase I protection assay. Increasing amounts of nuclear extract led to the protection of three distinct regions termed A, B, and C (Figure 4B, lanes 3 and 4). Sequences within these protected regions were found to overlap with several transcription factor-binding sites identified with the MatIspector program. In particular, protected region "C" overlaps with binding sites for two transcriptional repressor/activator proteins known as Barx2 and Engrailed-1, of which the former is abundantly expressed in HNSCC. Owing to the large size of the probe used in the DNase I protection assay, the probe actually overlapped sequences from the LEKTI promoter with both putative repressor and activator binding sites. Protected fragment A on the gel is actually found between -1382 to $-1398 \mathrm{bp}$, which occurs within the region expected to contain an enhancer (i.e., from 1359 to $1407 \mathrm{bp}$ ). Interestingly, the protected sequence from "A" overlaps with a v-Jun recognition site. We have subcloned the $48 \mathrm{bp}$ fragment from 1359 to $1407 \mathrm{bp}$ containing the $\mathrm{v}$-Jun recognition site and found that it indeed behaves as a transcriptional enhancer when cloned upstream of the thymidine kinase promoter and transfected into UMSCC-1 (data not shown). We have not tested the putative enhancer sequence transcription in NHEK but our results suggest that the sequence can activate transcription in UMSCC-1 in the absence of adjacent nucleotide sequences (which may recruit transcriptional repressors).

\section{Conclusion}

These data confirm our previous in vitro and in vivo findings that the loss of LEKTI expression in HNSCC results in a cellular phenotype with locally aggressive behavior. Our findings shed new light on mechanisms of PNI and LVI, offer potential prognostic value, and may bring insight to patient selection for therapeutic approaches.

\section{Acknowledgements}

\section{Funding}

Supported in part by the NIH-NCI P50 CA097007, NIH R01 DE013954, NIH P30 CA016672, Alando J. Ballantyne Distinguished Chair in Head and Neck Surgery award, Michael A. O'Bannon Endowment for Cancer Research, NIH INRS Award T32 CA060374, and AAO-HNSF Percy Memorial Grant.

\section{Author contributions}

A.J, T.S, M.F, and G.L.C provided intellectual input into the design and presentation of the study. A.J, M.J, and T.S wrote the manuscript. Y.H, Y.K., and T.S carried out experiments. K.J. and R.J. organized data and reviwed literature. R.P. participated in project discussion.

\section{Conflict of interest}

The author declares no conflict of interest.

\section{References}

1. Matrisian LM. Cancer biology: extracellular proteinases in malignancy. Curr Biol. 1999;9(20):R776-R778.

2. Doucet A, Overall CM. Protease proteomics: revealing protease in vivo functions using systems biology approaches. Mol Aspects Med. 2008;29(5):339-358.

3. Yadav L, Puri N, Rastogi V, et al. Matrix metalloproteinases and cancer - roles in threat and therapy. Asian Pac $J$ Cancer Prev 2014;15(3):1085-1091.

4. Fonovic M, Turk B. Cysteine cathepsins and extracellular matrix degradation. Biochim Biophys Acta. 2014;1840(8):2560-2570.

5. Davies KJ. The Complex Interaction of Matrix Metalloproteinases in the Migration of Cancer Cells through Breast Tissue Stroma. Int J Breast Cancer. 2014.

6. Shen $\mathrm{W}, \mathrm{Xi} \mathrm{H}, \mathrm{Wei} \mathrm{B}$, et al. The prognostic role of matrix metalloproteinase 2 in gastric cancer: a systematic review with meta-analysis. J Cancer Res Clin Oncol. 2014;140(6):1003-1009.

7. Pemberton PA, Tipton AR, Pavloff N, et al. Maspin is an intracellular serpin that partitions into secretory vesicles and is present at the cell surface. J Histochem Cytochem. 1997;45(12):1697-1706.

8. Toth M, Bernardo MM, Gervasi DC, et al. Tissue inhibitor of metalloproteinase (TIMP)-2 acts synergistically with synthetic matrix metalloproteinase (MMP) inhibitors but not with TIMP-4 to enhance the (Membrane type 1)-MMP-dependent activation of pro-MMP-2. J Biol Chem. 2000;275(75):41415-4123. 
9. Silverman GA, Bird PI, Carrell RW, et al. The serpins are an expanding superfamily of structurally similar but functionally diverse proteins. Evolution, mechanism of inhibition, novel functions, and a revised nomenclature. J Biol Chem. 2001;276(75):33293-33296.

10. Jayakumar A, Kang Y, Frederick MJ, et al. Inhibition of the cysteine proteinases cathepsins $\mathrm{K}$ and $\mathrm{L}$ by the serpin headpin (SERPINB13): a kinetic analysis. Archives of Biochemistry \& Biophysics. 2003;409(2):367-374.

11. Roelandt T, Thys B, Heughebaert C, et al. LEKTI-1 in sickness and in health. Int J Cosmet Sci. 2009;31(4):247-254.

12. Khokha R, Murthy A, Weiss A. Metalloproteinases and their natural inhibitors in inflammation and immunity. Nat Rev Immunol. 2013;13(9):649-665.

13. Gong Y, Chippada-Venkata UD, Oh WK. Roles of matrix metalloproteinases and their natural inhibitors in prostate cancer progression. Cancers. 2014;6(3):1298-1327.

14. Coussens LM, Werb Z. Matrix metalloproteinases and the development of cancer. Chem \& Bio. 1996;3:895-904.

15. Werb Z, Vu TH, Rinkenberger JL, et al. Matrix-degrading proteases and angiogenesis during development and tumor formation. APMIS. 1999; 107:11-18

16. Maass N, Hojo T, Zhang M, et al. Maspin--a novel protease inhibitor with tumor-suppressing activity in breast cancer. Acta Oncol. 2000;39(8):931-934.

17. Maass N, Nagasaki K, Ziebart M, et al. Expression and regulation of tumor suppressor gene maspin in breast cancer. Clin Breast Cancer. 2002;3(4):281-287.

18. Zou Z, Zhang W, Young D, et al. Maspin expression profile in human prostate cancer $(\mathrm{CaP})$ and in vitro induction of Maspin expression by androgen ablation. Clin Cancer Res. 2002;8(5):1172-1177.

19. Ortega N, Behonick D, Stickens D, et al. How proteases regulate bone morphogenesis. Ann N Y Acad Sci. 2003;995:109-116.

20. Woessner JF Jr. MMPs and TIMPs--an historical perspective. Mol Biotechnol. 2002;22(1):33-49.

21. Bausch D, Pausch T, Krauss T, et al. Neutrophil granulocyte derived MMP-9 is a VEGF independent functional component of the angiogenic switch in pancreatic ductal adenocarcinoma. Angiogenesis. 2011;14(3):235-243

22. Bennett K, Heywood W, Di WL, et al. The identification of a new role for LEKTI in the skin: The use of protein 'bait' arrays to detect defective trafficking of dermcidin in the skin of patients with Netherton syndrome. J Proteomics. 2011;75(13):3925-3937.

23. Gonzalez HE, Gujrati M, Frederick M, et al. Identification of 9 genes differentially expressed in head and neck squamous cell carcinoma. Arch Otolaryngol Head Neck Surg. 2003;129(7):754-759.

24. Shah TM, Bhatt VD, Tripathi AK, et al. The landscape of alternative splicing in buccal mucosa squamous cell carcinoma. Oral Oncol. 2013;49(6):604-610.

25. Chavanas S, Bodemer C, Rochat A, et al. Mutations in SPINK5, encoding a serine protease inhibitor, cause Netherton syndrome. Nat Genet. 2000;25(2):141-142.

26. Sprecher E, Chavanas S, DiGiovanna JJ, et al. The spectrum of pathogenic mutations in SPINK5 in 19 families with Netherton syndrome: implications for mutation detection and first case of prenatal diagnosis. J Invest Dermatol. 2001;117(2):179-187.

27. Muller FB, Hausser I, Berg D, et al. Genetic analysis of a severe case of Netherton syndrome and application for prenatal testing. Br J Dermatol. 2002;146(3):495-499.
28. Bitoun E, Bodemer C, Amiel J, et al. Prenatal diagnosis of a lethal form of Netherton syndrome by SPINK5 mutation analysis. Prenat Diagn. 2002;22(2):121-126.

29. Komatsu N, Takata M, Otsuki N, et al. Elevated stratum corneum hydrolytic activity in Netherton syndrome suggests an inhibitory regulation of desquamation by SPINK5-derived peptides. $J$ Invest Dermatol. 2002;118(3):436-443.

30. Bitoun E, Chavanas S, Irvine AD, et al. Netherton syndrome: disease expression and spectrum of SPINK5 mutations in 21 families. J Invest Dermatol. 2003;118(2):352-361.

31. Raghunath $\mathrm{M}$, Tontsidou L, Oji V, et al.SPINK5 and Netherton syndrome: novel mutations, demonstration of missing LEKTI, and differential expression of transglutaminases. J Invest Dermatol. 2004;123(3):474s483.

32. Descargues P, Deraison C, Bonnart C, et al. Spink5-deficient mice mimic Netherton syndrome through degradation of desmoglein 1 by epidermal protease hyperactivity. Nat Genet. 2005;37(1):56-65.

33. Komatsu N, Saijoh K, Jayakumar A, et al. Correlation between SPINK5 gene mutations and clinical manifestations in Netherton syndrome patients. J Invest Dermatol. 2008;128(5):1148-1159.

34. Di WL, Hennekam RC, Callard RE, et al. A heterozygous null mutation combined with the G1258A polymorphism of SPINK5 causes impaired LEKTI function and abnormal expression of skin barrier proteins. $\mathrm{Br} J$ Dermatol. 2009;161(2):404-412.

35. Diociaiuti A, Castiglia D, Fortugno P, et al. Lethal Netherton syndrome due to homozygous p.Arg371X mutation in SPINK5. Pediatr Dermatol. 2013;30(4):e65-e67.

36. D'Alessio M, Fortugno P, Zambruno G, et al. Netherton syndrome and its multifaceted defective protein LEKTI. G Ital Dermatol Venereol. 2013;148(1):37-51.

37. Wang S, Olt S, Schoefmann N, et al. SPINK5 knockdown in organotypic human skin culture as a model system for Netherton syndrome: effect of genetic inhibition of serine proteases kallikrein 5 and kallikrein 7. Exp Dermatol. 2014;23(7):524-526.

38. Furio L, Hovnanian A. Netherton syndrome: defective kallikrein inhibition in the skin leads to skin inflammation and allergy. Biol Chem. 2014;395(9):945-958.

39. Itoh K, Kako T, Suzuki N, et al. Severe lethal phenotype of a Japanese case of Netherton syndrome with homozygous founder mutations of SPINK5 c.375_376delAT. J Dermatol. 2015.

40. Nair J, Jain P, Chandola U, et al. Gene and miRNA expression changes in squamous cell carcinoma of larynx and hypopharynx. Genes Cancer. 2015;6(7-8):328-340.

41. Dal MA, Fortugno P, Donadon I, et al. Exon-Specific U1s Correct SPINK5 Exon 11 Skipping Caused by a Synonymous Substitution that Affects a Bifunctional Splicing Regulatory Element. Hum Mutat. 2015;36(5):504-512.

42. Mitsudo K, Jayakumar A, Henderson Y, et al. Inhibition of serine proteinases plasmin, trypsin, subtilisin $\mathrm{A}$, cathepsin $\mathrm{G}$, and elastase by LEKTI: a kinetic analysis. Biochemistry. 2003;42(13):3874-3881.

43. Jayakumar A, Kang Y, Mitsudo K, et al. Expression of LEKTI domains 6-9' in the baculovirus expression system: recombinant LEKTI domains 6-9' inhibit trypsin and subtilisin A. Protein Expr Purif. 2004;35(1):93-101.

44. Jayakumar A, Kang Y, Henderson Y, et al. Consequences of C-terminal domains and N-terminal signal peptide deletions on LEKTI secretion, stability, and subcellular distribution. Arch Biochem Biophys. 2005;435(1):89-102. 
45. Schechter NM, Choi EJ, Wang ZM, et al. Inhibition of human kallikreins 5 and 7 by the serine protease inhibitor lympho-epithelial Kazal-type inhibitor (LEKTI). Biol Chem. 2005;386(11):1173-1184.

46. Deraison C, Bonnart C, Lopez F, et al. LEKTI fragments specifically inhibit KLK5, KLK7, and KLK14 and control desquamation through a pH-dependent interaction. Mol Biol Cell. 2007;18(9):3607-3619.

47. Borgono CA, Michael IP, Komatsu N, et al. A potential role for multiple tissue kallikrein serine proteases in epidermal desquamation. $J$ Biol Chem. 2007;282(6):3640-3652.

48. Jayakumar A, Chattopadhyay C, Wu HK, et al. LEKTI, A Physiological Inhibitor of Multiple Serine Proteinases, Blocks Migration and Invasion of Head and Neck Squamous Cell Carcinoma (HNSCC) Cells. MOJ Proteomics Bioinform. 2014:1(3).
49. Jayakumar A, Wu HK, Briggs K, et al. LEKTI, A Physiological Inhibitor of Multiple Serine Proteinases, Suppresses Perineural and Lymphovascular Invasion of Human Head and Neck Cancer Cells in A Mouse Orthotopic Model. MOJ Proteomics Bioinform. 2014:1(5).

50. Frommer M, McDonald LE, Millar DS, et al. A genomic sequencing protocol that yields a positive display of 5-methylcytosine residues in individual DNA strands. Proc Natl Acad Sci USA. 1992;89(5):1827-1831. 